\title{
Automated Analysis of Aggregated Datasets to Identify Climatic Predictors of Botrytis Bunch Rot in Wine Grapes
}

\author{
Gareth N. Hill,† Robert M. Beresford, and Katherine J. Evans
}

First and second authors: The New Zealand Institute for Plant \& Food Research Limited, Mt Albert Research Centre, Auckland Mail Centre, Auckland 1142, New Zealand; and third author: Tasmanian Institute of Agriculture, University of Tasmania, Private Bag 98, Hobart, Tasmania 7001, Australia.

Accepted for publication 25 June 2018.

\begin{abstract}
Botrytis bunch rot (BBR), caused by Botrytis cinerea, results in serious losses to wine-grape production in some seasons during the preharvest period. In order to predict seasons that are at risk from BBR, datasets consisting of 25 disease, weather and vine phenology variables were aggregated from 101 SiteYears across seven regions and nine growing seasons. Automated analyses were used to compare a range of statistical methods for their ability to predict BBR epidemics, including the Kruskal-Wallis test, logistic regression, receiver operating characteristic analysis, and skill-scores. Variables based on relative humidity and surface-wetness duration were significant and consistent predictors of

BBR epidemics across the range of analyses applied. Variables integrating temperature and wetness duration, including the Bacchus and Broome models, also demonstrated high predictive ability; however, they did not outperform their constituent components in all analyses. Automation of data analyses was an effective way to compare a wide range of statistical methods and a large number of variables with minimal user input, following initial code development. Significant time was needed to check input data and software code, but a greater return on investment would occur should the analytical process be applied to new datasets, including those from other pathosystems.
\end{abstract}

Botrytis bunch rot (BBR) in wine grapes, caused by the fungus Botrytis cinerea, is a major economic disease for grape growers worldwide that is heavily influenced by seasonal weather patterns. The pathogen can infect via airborne conidia throughout flower and fruit development and can remain latent until symptom expression late in the growing season (Hill et al. 2014). Infection of grape tissue by $B$. cinerea requires moisture and wet growing seasons typically result in more severe BBR epidemics (Coertze et al. 2001; Edwards et al. 2009).Various studies have described environmental conditions favoring the germination of $B$. cinerea conidia or infection of grapevine tissues based on results of in vitro or controlled-environment experiments (Broome et al. 1995; Ciliberti et al. 2014; GonzálezDomínguez et al. 2015; Nair and Allen 1993; Nelson 1951b; Kim et al. 2007). Empirical models reported in these studies comprise relatively simple equations, combining variables based on temperature, relative humidity and surface-wetness duration into risk indices that provide a simple numerical representation of the suitability for $B$. cinerea infection. Risk indices can be defined more generally as, "formalized algorithms that assess disease risk factors to inform the need for crop protection" (Gent et al. 2013).

There have been attempts to take a more season-wide view of a BBR epidemic, rather than identifying brief periods of weather suitable for $B$. cinerea growth. Beresford et al. (2012) incorporated the Bacchus algorithm (Kim et al. 2007), which relates $B$. cinerea growth to temperature and surface wetness, to describe the temporal progression of BBR risk throughout the season via a decision support system (DSS) (Gent et al. 2013; Shtienberg 2013). In contrast to the empirical approach employed in the DSS, González-Domínguez et al. (2015) developed a mechanistic model incorporating all the stages of the BBR disease cycle. Despite these developments, the

†Corresponding author: G. N. Hill; E-mail: gareth.hill@plantandfood.co.nz

*The $\boldsymbol{e}$-Xtra logo stands for "electronic extra" and indicates that one supplementary figure and five supplementary files are published online.

(c) 2019 The American Phytopathological Society main functional components of both the DSS and mechanistic model are existing weather risk models with the same predictive capabilities as those risk models. There are many other weather variables, combinations of weather variables, and derivatives of weather variables that may offer improved predictive ability. Time constraints resulting from manual data analysis methods have meant many of these weather variables have not been adequately investigated.

Increasing computing power is providing the means to capture and process abundant data, and to reveal associations among weather variables that describe the weather-pathogen-host interaction. The spatial and temporal resolution of weather data are also increasing due to accessibility of weather sensors and data loggers, improved telecommunications technologies, and interpolated weather grids (Mourtzinis et al. 2017). Automation of data analyses can potentially increase the efficiency, volume of data, and number of variables explored during studies of plant disease epidemiology, as well as revealing the influence of a large number of weather variables and their interactions on disease progression. Meta-analysis and data mining are more advanced applications of data automation that have been used in a disease management context and for investigation of treatment effects (Scherm et al. 2014).

Automation of data analyses means that a wide range of statistical approaches to identifying weather-related predictors of plant diseases can be applied in a single study. The key benefit of automation is that different analyses can be investigated quickly and easily, without having to rely on the results of a single approach that may not provide all the necessary information. Potential statistical methods not yet applied to BBR in wine grapes include the Kruskal-Wallis test, receiver operating characteristic (ROC) analysis, multivariable logistic regression, skill-score analysis and window-pane analysis. The key features of these methods are described next, noting that the results of some of these analyses provide similar or related insights on the relationships between variables.

Quickly identifying weather variables with predictive ability can be done by statistically comparing populations where a disease epidemic either did or did not occur, which can be done using 
Student's $t$ test (Student 1908) if the weather variable data are normally distributed. However, nonparametric tests, such as the Kruskal-Wallis test (Kruskal and Wallis 1952) or Mann-Whitney $U$ test (Mann and Whitney 1947), can be advantageous in automated analyses as they can be used to compare the distributions of data that are not normally distributed, allowing the inclusion of data without the need for normality checking or transformation.

Determining specific weather variable thresholds for predicting a disease outcome can be done using ROC (Greiner et al. 2000; Zweig and Campbell 1993) analysis, which evaluates various discrimination thresholds for a given outcome and results in an ROC curve that allows the optimum threshold to be identified. The area under the curve (AUC) of the ROC curve provides a measure of the overall accuracy with which an indicator variable discriminates between positive and negative outcomes (Hanley and McNeil 1982). This type of analysis has been used in epidemiological studies of a number of plant diseases, including Sclerotinia stem rot in oil seed rape (Yuen et al. 1996), apple scab, Fusarium head blight in wheat (Shah et al. 2013), elsinoe spot in apples (Beresford et al. 2015), and kiwifruit bacterial canker (Beresford et al. 2016).

Once a predictor variable and discriminatory threshold have been identified, skill score analysis can be used to evaluate the predictive ability of the variable and provide a numeric summary of the probability of agreement between the predicted and actual outcomes beyond that expected due to chance (Hughes 2012). The two most commonly used skill scores are the Heidke skill score (Heidke 1926), applied in meteorology to evaluate weather forecasts, and Cohen's kappa (Cohen 1960), applied in psychology and similar fields. Despite the scores having different names and equations, the resulting skill scores are identical (Hyvarinen 2014).

Identifying the predictive ability of multiple independent weather variables, can be done using multivariable logistic regression, which can use either continuous or binary data to predict a binary dependent variable, e.g., epidemic or no epidemic (Cox 1958), and can include weather variables separately or combined in a single regression. This type of analysis has been used to study the epidemiology of a number of plant diseases, including Sclerotinia stem rot in oil seed rape (Yuen et al. 1996), bacterial canker in kiwifruit (Froud 2015), and Fusarium head blight in wheat (Shah et al. 2013).
Testing the predictive ability of a weather variable at different times in the season can be done using window-pane analysis, which examines a weather variable that is summarized over a specific time interval for changes in its relationship to disease outcomes as the time interval is moved sequentially throughout a season. The weather variable has maximum predictive ability in the part of the season when it is most highly correlated with disease. When different weather variables are compared in this way, the best predictor, and its timing, can be identified. The primary advantage of window-pane analysis is that it considers the seasonality of both weather and disease, allowing identification of relationships between weather, disease and time. Automated data analyses were applied during window-pane analysis of Septoria tritici blotch of wheat (Coakley et al. 1985) because this analytical method is more computationally-intensive than whole season or single time-period analyses. The window-pane approach has since been used to study the epidemiology of other plant diseases, including Fusarium head blight and brown rust of wheat (Gouache et al. 2015; Kriss et al. 2010) and European canker of apple (Beresford and Kim 2011).

The purpose of this study was to identify weather-based predictors of BBR epidemics using data automation techniques to analyze a dataset aggregated from multiple wine production regions in New Zealand and southeast Australia over multiple seasons. The data were collected during a number of BBR management and epidemiology studies. The objectives of the current study were to develop generalized data automation procedures to compare the usefulness of various statistical methods for identifying weather-based predictors of BBR severity at harvest. Application of data automation to other datasets with minimal user input in future studies is discussed.

\section{MATERIALS AND METHODS}

Vineyard site and year (SiteYear) data. Data were obtained from nontreated plots of 101 trials in plantings of Vitis vinifera managed according to local viticultural practices for the production of white table wine. Trials were conducted in five regions of New Zealand and two regions of south-eastern Australia across nine growing seasons (Fig. 1; Table 1). The varieties of $V$. vinifera were Chardonnay $(n=37)$, Gewürztraminer $(n=1)$, Pinot gris $(n=2)$, Riesling $(n=5)$, Sauvignon

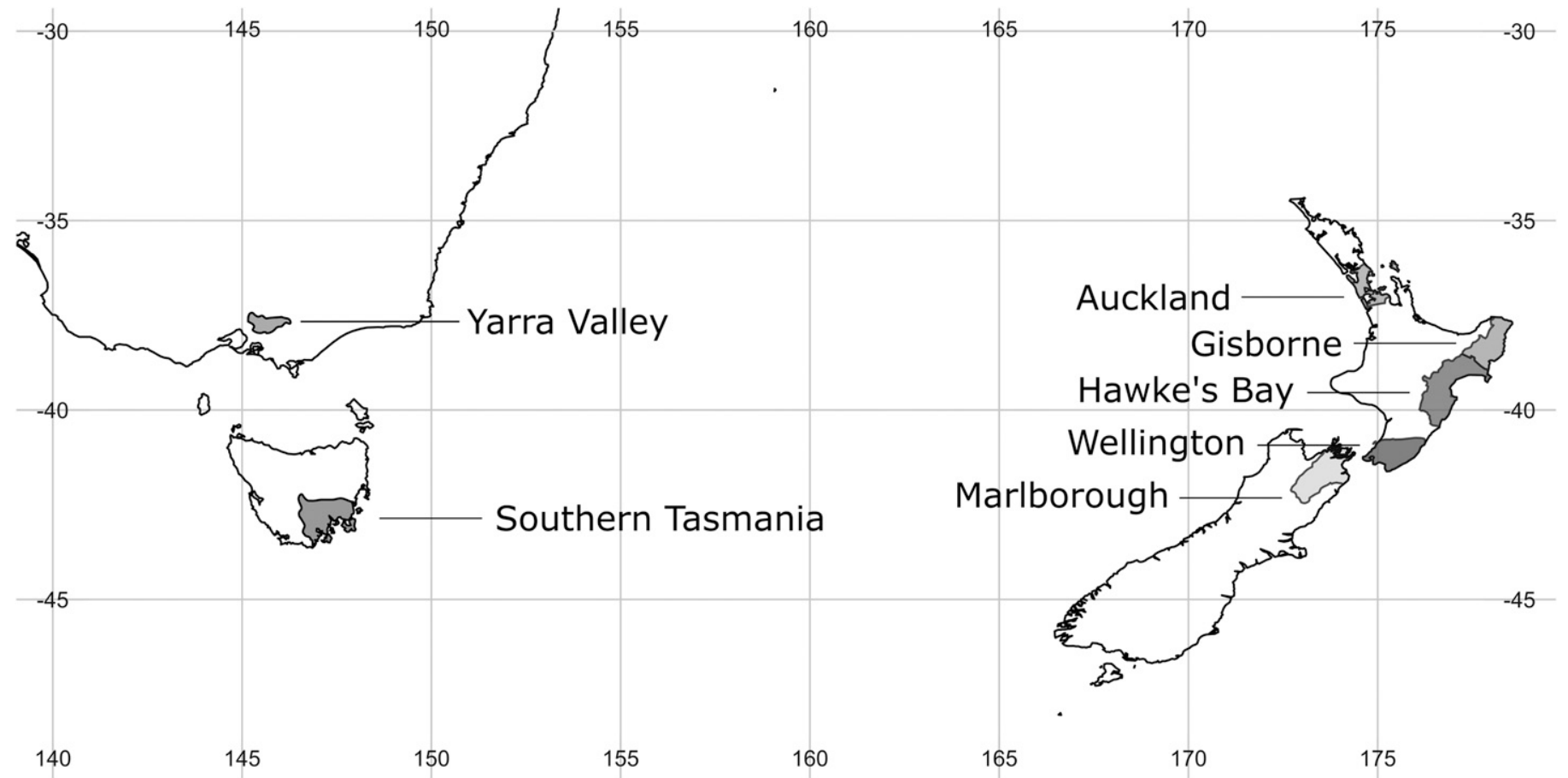

Fig. 1. Regions in Australia and New Zealand (shaded) from which historical data were collected. 
blanc ( $n=53)$, Sémillon $(n=2)$, and Verdelho $(n=1)$. The GPS coordinates of each trial site and two key vine phenology dates were recorded: flowering (E-L 19) (Coombe 1995) and véraison (E-L 35). The small-plot, replicated trials from which these data were obtained varied in the type and number of treatments; however, all trials were a randomized complete-block design, and standard methods for disease severity assessments were applied.

The response variable for all analyses was the severity of BBR at harvest, assessed using the visual estimation method (Hill et al. 2010). The distribution of harvest BBR severity for all SiteYears was skewed toward the lower severities (Fig. 2), with a median severity of $4.3 \%$, a mean severity of $7.7 \%$, and a range of 0.0 to $40.0 \%$.

Weather data. Weather data were collected from temperature, relative humidity $(\mathrm{RH})$, and rainfall sensors from various manufacturers that were attached to electronic data loggers and kept appropriately calibrated. For example, Campbell Scientific sensors (Logan, UT) were used at all New Zealand sites. Data loggers recorded averaged or summed data for any given hour. Unless stated otherwise, the surface wetness value from the electrical resistance sensors for any given hour was considered 'wet' when the mean reading of the leaf-wetness sensor was $>50 \%$ of the maximum response.

Data were obtained from sensors housed in temporary on-vineyard weather stations for the Australian trial sites, or from the nearest weather station available through MetWatch Online (HortPlus (NZ) Ltd., Hastings, New Zealand) in New Zealand. Weather stations were all within $10 \mathrm{~km}$ of the trial sites, with a median distance of $0.97 \mathrm{~km}$. Distances were calculated by accessing a list of all available weather stations in New Zealand and applying the Vincenty (ellipsoid) method via the geosphere R package (Hijmans 2016) to the GPS coordinates for weather stations and trial sites.

All sensors were deployed as per World Meteorological Organization (WMO) guidelines (World Meteorological Organization 2014), with sensor manufacturer varying among sites. Leaf-wetness sensors, which lack a WMO guideline, were unpainted electrical resistance grids (237-L; Campbell Scientific) placed at a height of $1.5 \mathrm{~m}$ and set at an angle of approximately $20^{\circ}$.

Software and code. Raw trial data were arranged using Microsoft Excel 2013 (version 15.0.4763; Microsoft, Redmond, WA). All code was written and executed in R Studio (version 1.0.44; R Studio Team 2016) running version 3.3.2 of R (R Core Team 2016). $R$ packages used in the analyses included: 'pROC' using default settings for ROC analysis (Robin et al. 2011), 'glmulti' using default settings for logistic regression (Calcagno 2013), 'ggplot2' for graphics (Wickham 2009), 'tidyverse' for data manipulation (Wickham 2016), 'geosphere' for distance calculations (Hijmans 2016), 'SimpleMappr' to generate the regional map (Shorthouse 2017), and 'knitr' for general formatting and graphics generation (Xie 2016).

The code required the following inputs, supplied as commaseparated value (CSV) files: SiteYear information (GPS coordinates, vine phenology dates and BBR severity), weather station information (station code, GPS coordinates) and hourly weather data (station code, temperature, RH, rainfall and surface wetness duration). During development, the code was executed in sections and adjusted to correct errors and ensure analyses were computationally and statistically sound. The final version of the entire code was able to be executed in its entirety and all analyses carried out with no further user input, resulting in an HTML file that contained all data summaries, tables, and figures (Supplementary Files S1, S2, S3, S4, and S5; Supplementary Fig. S1).

Case definition. A threshold of $3 \%$ BBR severity at harvest is often used by wine producers for negotiations about grape price, particularly in Marlborough, New Zealand's largest wine producing region (Andrew and Lupton 2013; Evans 2013). Therefore, each trial was assigned a value of 1 ("case"), if BBR severity was greater than or equal to $3 \%$ at harvest, or otherwise, a value of 0 ("control"). There were 56 cases and 45 controls.

Weather variables. Weather variables (Table 2 ) were chosen from those previously used for BBR epidemic prediction and from others with a proven or perceived influence on Botrytis cinerea, including the time between one vine phenology stage and another (Days and Hours), hereafter referred to as 'season length'. Timedependent variables were defined as those that involved 'hours' or 'days' as units of measure (e.g., thermal time defined below).

The period over which each variable was calculated depended on the type of analysis. Unless stated otherwise, weather variables were calculated between the site-specific crop stages of flowering (E-L 19) and harvest (E-L 38). Hourly weather variables were either summed for a given time period ("total"; _Tot), averaged for a given time period ("mean"; _Mean), or summed for a given time period

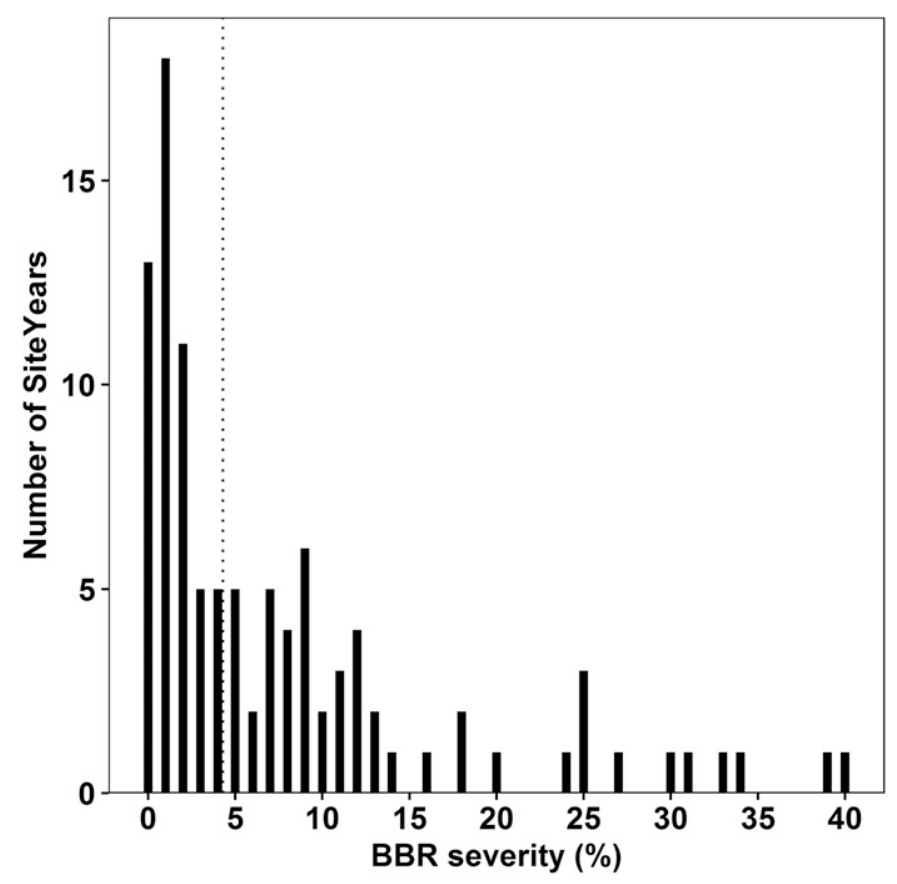

Fig. 2. Distribution of Botrytis bunch rot (BBR) severities at harvest for all SiteYears. The dotted line indicates median BBR severity $(4.3 \%)$.

TABLE 1. Number of trials in each region (Fig. 1) and season from which data for grapevine phenology and severity of Botrytis bunch rot at harvest were collected

\begin{tabular}{|c|c|c|c|c|c|c|c|c|}
\hline Season & Auckland & Gisborne & Hawke's Bay & Marlborough & Wellington & Southern Tasmania & Yarra Valley & Season total \\
\hline 2000/01 & 1 & 0 & 1 & 1 & 0 & 0 & 0 & 3 \\
\hline 2001/02 & 1 & 0 & 1 & 1 & 0 & 0 & 0 & 3 \\
\hline $2002 / 03$ & 1 & 0 & 6 & 6 & 0 & 0 & 0 & 13 \\
\hline $2006 / 07$ & 1 & 0 & 2 & 2 & 0 & 2 & 3 & 10 \\
\hline 2007/08 & 1 & 0 & 10 & 2 & 0 & 4 & 3 & 20 \\
\hline 2008/09 & 1 & 4 & 9 & 1 & 0 & 2 & 3 & 20 \\
\hline $2009 / 10$ & 0 & 5 & 8 & 0 & 0 & 0 & 0 & 13 \\
\hline $2011 / 12$ & 0 & 4 & 9 & 4 & 1 & 0 & 0 & 18 \\
\hline $2012 / 13$ & 1 & 0 & 0 & 0 & 0 & 0 & 0 & 1 \\
\hline Region total & 7 & 13 & 46 & 17 & 1 & 8 & 9 & 101 \\
\hline
\end{tabular}


and divided by the number of days in that period ("mean daily"; _MD).

Additional weather variables were derived from individual weather variables to assess their potential for predicting BBR severity at harvest. Temperature was used to derive thermal time, calculated using a base temperature of $0^{\circ} \mathrm{C}$ (ThermTime 0$)$ or $10^{\circ} \mathrm{C}$ (ThermTime10), with mean daily temperature calculated from hourly average temperatures. ThermTime10 is the equivalent of what is commonly referred to as 'growing degree days' or GDD. RH was used to determine the number of hours in a day in which the RH was higher than a threshold and then averaged (RHHoursMD) or summed (RHHoursTot) for a given period. Rainfall was calculated as the total (RainTot) and mean daily (RainMD) values for a given period. Total rainfall was also calculated for the last fortnight before harvest (RainLF), or for 14 days prior to the end of any given date in the window-pane analyses. The former was based on a perception of grape growers that rainfall during this period leads to a higher severity of BBR severity at harvest than dry weather.

Thresholds used in the calculation of surface wetness and thermal time can vary, and common thresholds can be arbitrary. Therefore, the Kruskal-Wallis test (Kruskal and Wallis 1952) was used to determine optimal thresholds for these variables by calculating the variables using a range of thresholds and identifying the threshold that gave the largest significant difference between cases and controls. The optimal wetness sensor threshold for hourly leaf wetness observations was obtained by varying the threshold between 0 and $100 \%$. Similarly, the optimal base temperature for calculating thermal time was obtained by varying the threshold from 0 to $30^{\circ} \mathrm{C}$. Two extra variables were thus created using those optimal thresholds: WetThrshMD and ThermTimeThrsh. Ten SiteYears were excluded from the optimal wetness threshold determination because leaf-wetness observations were binary ( 0 or 100); however, these Site Years were included in all other analyses. The Kruskal-Wallis test was also used to identify an optimal RH threshold for determining number of hours above that threshold for any given day for the two weather variables RHHoursMD and RHHoursTot.

Two models describing infection risk were included, Bacchus (Kim et al. 2007) and Broome model (Broome et al. 1995). While both of these risk models typically have a set of rules that translate into risk periods, only the underlying equation was used to calculate a risk value for each wet hour (leaf wetness $>50$ ) in order for the raw risk value to be manipulated mathematically. The Bacchus risk value was calculated as follows:

$$
x=1 /\left(\left(84.37-(7.238 T)+\left(0.156 T^{2}\right)\right)\right.
$$

where $x=$ the Bacchus risk value for a given wet hour (using a threshold of $50 \%$ surface wetness), and $T=$ mean temperature. This equation represents a correction to the model published by Kim et al. (2007).

The Broome risk value was calculated as follows:

$$
x=-2.647866-0.374927 W+0.061601 W T-0.001511 W T^{2}
$$

where $x=$ Broome risk for a given wet period, $W=$ duration of surface wetness (h) and $T=$ mean temperature during the period of surface wetness. In this study, Broome risk was calculated hourly and therefore only when $W=1$; hence, the original equation was modified to remove $W$. The intercept was also removed so that the value of $x$ was never negative. The modified Broome risk value was calculated as follows:

$$
x=0.00616 T-0.00015 T^{2}
$$

where $x=$ Broome risk value for a given wet hour, and $T=$ mean temperature. Equation 1 and Equation 3 are compared in Figure 3.

Both Bacchus and Broome risk values were also calculated using leaf wetness with a $4 \mathrm{~h}$ dryout period taken into account (BacchusDryout and BroomeDryout), as this is common in the implementation of disease risk models in DSSs (Beresford and Spink 1992). If there are four or fewer dry hours between two wet hours, then those dry hours are considered to be wet for the purposes of the risk-value calculations.

Distribution of weather variables. To compare the distributions of weather variables, the values of each were normalized to a range of 0 to 1 , as follows:

$$
f(x)=\left(x-x_{\min }\right) /\left(x_{\max }-x_{\min }\right)
$$

where $x=$ weather variable value for a given SiteYear, $x_{\min }=$ minimum value across all SiteYears and $x_{\max }=$ maximum value

\begin{tabular}{|c|c|c|c|c|}
\hline ID code & Weather variable & Input 1 & Input 2 & Input 3 \\
\hline Days & Days (total) & Time & - & - \\
\hline Hours & Hours (total) & Time & - & - \\
\hline TempMean & Temperature (mean) & Temperature & - & - \\
\hline TempMin & Temperature (minimum) & Temperature & - & - \\
\hline TempMax & Temperature (maximum) & Temperature & - & - \\
\hline ThermTime & Thermal time; $0^{\circ} \mathrm{C}$ threshold & Temperature & Time & - \\
\hline ThermTime10 & Thermal time; $10^{\circ} \mathrm{C}$ threshold & Temperature & Time & - \\
\hline ThermTimeThrsh & Thermal time; optimized threshold & Temperature & Time & - \\
\hline WetMD & Wet hours (mean daily) & Wetness & - & - \\
\hline WetTot & Wet hours (total) & Wetness & Time & - \\
\hline WetThrshMD & Wet hours (mean daily); optimized threshold & Wetness & - & - \\
\hline RHMean & RH (mean) & RH & - & - \\
\hline RHHoursMD & RH hours (mean daily) & RH & - & - \\
\hline RHHoursTot & RH hours (total) & RH & Time & - \\
\hline RainMD & Rainfall (mean daily) & Rain & - & - \\
\hline RainTot & Rainfall (total) & Rain & Time & - \\
\hline RainLF & Rainfall (last fortnight) & Rain & - & - \\
\hline BacchusMD & Bacchus risk (mean daily) & Wetness & Temperature & - \\
\hline BacchusTot & Bacchus risk (total) & Wetness & Temperature & Time \\
\hline BacchusDryoutMD & Bacchus risk with dryout (mean daily) & Wetness & Temperature & - \\
\hline BacchusDryoutTot & Bacchus risk with dryout (total) & Wetness & Temperature & Time \\
\hline BroomeMD & Broome risk (mean daily) & Wetness & Temperature & - \\
\hline BroomeTot & Broome risk (total) & Wetness & Temperature & Time \\
\hline BroomeDryoutMD & Broome risk with dryout (mean daily) & Wetness & Temperature & - \\
\hline BroomeDryoutTot & Broome risk with dryout (total) & Wetness & Temperature & Time \\
\hline
\end{tabular}
across all SiteYears. Normalized values were only used to compare distributions and raw values were used in all other analyses.

TABLE 2. Weather variables calculated from raw weather data, ID code, and raw data inputs used to calculate each variable

a The time period over which variables were calculated differed depending on the analysis. 
Case/control comparisons and logistic regression. Initial screening of all weather variables was done using the nonparametric Kruskal-Wallis test to compare cases and controls, as not all the weather variables had normal distributions. Statistical differences between cases and controls for each variable were identified through the $P$ value. Nontransformed data were used in all comparisons. If season length (Days or Hours), which differed between SiteYears, was found to be significant following the initial case/ control comparisons, then all other time-dependent variables were excluded from further analyses to prevent collinearity with the time component.

Multivariable logistic regression was carried out using all possible combinations of two or more variables; single variable models were not used as these would have given similar, if not the same, results as the other univariate analyses. All non-timedependent weather variables were included in the analysis. Raw weather variable data were used, as well as a binary version of the data where each value was converted to a 0 or 1 using the individual weather variable thresholds calculated using ROC analysis $(0<$ threshold, $1 \geq$ threshold). Models were excluded if the weather variables were calculated from the same inputs and were therefore collinear (Table 2). The relative predictive quality of the models was measured using the Akaike information criterion (AIC) (Akaike 1974).

Receiver operating characteristic analysis and skill score testing. Additional information for each weather variable was obtained using ROC analysis. In each ROC analysis, the true positive proportion (TPP) and false positive proportion (FPP) were plotted for a range of threshold values to identify the optimum threshold for case/control prediction. The optimum threshold is that which maximizes the proportion of true positives and minimizes the proportion of false positives (Fig. 4). The discriminatory power of each weather variable was obtained in the form of the area under the ROC curve (AUC) (Hanley and McNeil 1982) and the weather variables were ranked based on Youden's index $(J)$, which is the difference between TPP and FPP and denotes the performance of the analysis (Youden 1950). The Wilcoxon test (Wilcoxon 1945) was used to test the significance of the AUC.

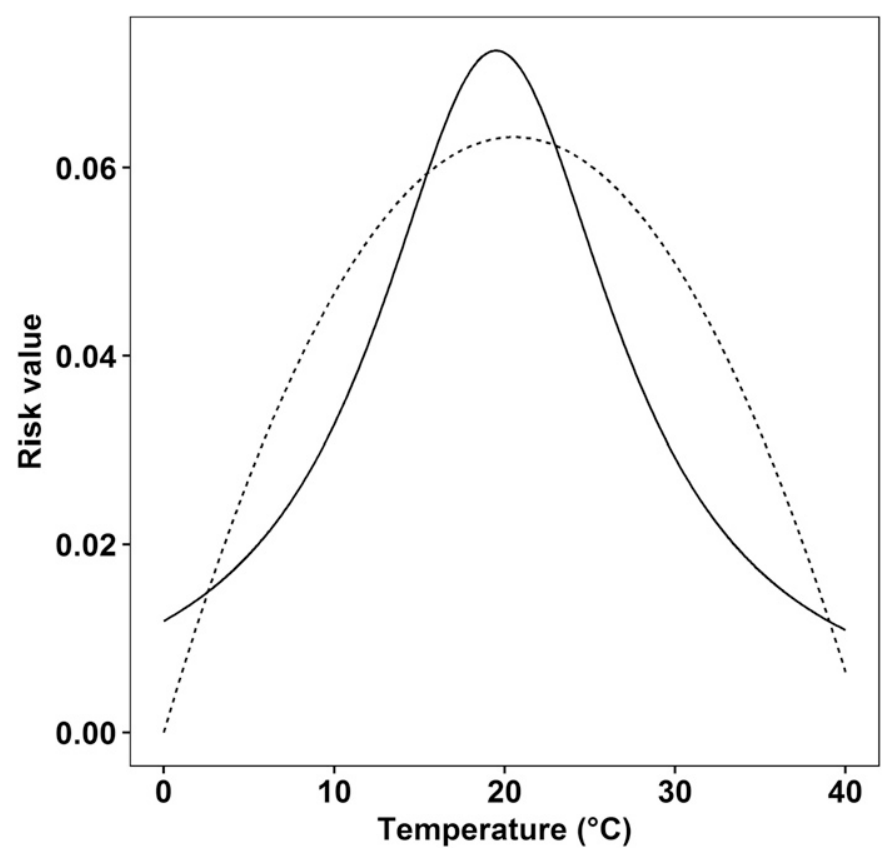

Fig. 3. Comparison of risk values generated from the Bacchus model (solid line; $\left.y=1 /\left(84.37-(7.238 x)+\left(0.156 x^{2}\right)\right)\right)$ and the modified Broome model (dashed line; $y=0.00616 x-0.00015 x^{2}$ ) across a range of mean temperature during any given hour of surface wetness.
In the absence of an external dataset to validate the predictive ability of the weather variables and the thresholds identified through ROC analysis, cross-validation was used. One thousand random combinations of SiteYears were generated for cross-validation, with each combination of SiteYears being split into one of two subsets: 67 random SiteYears in the 'training' subset and 34 random SiteYears in the 'validation' subset. For each combination, the training subset was used to calculate weather variable thresholds using ROC analysis, which were then used to predict the epidemic status of the validation SiteYears. A skill score in the form of Cohen's kappa statistic ( $\kappa)$ (Cohen 1960) was then calculated as follows:

$$
\kappa=2 \times\left(t_{p} t_{n}-f_{p} f_{n}\right) /\left(\left(t_{p}+f_{n}\right)\left(f_{n}+t_{n}\right)+\left(t_{p}+f_{p}\right)\left(f_{p}+t_{n}\right)\right)
$$

where $t_{p}=$ number of correctly predicted cases (true positives), $t_{n}=$ number of correctly predicted controls (true negatives), $f_{p}=$ number of wrongly predicted cases (false positives) and $f_{n}=$ number of wrongly predicted controls (false negatives). The mean threshold and $\kappa$ for all weather variables was calculated across all 1,000 SiteYear combinations along with the $95 \%$ confidence intervals. This equation is an expanded form of the original Cohen's kappa statistic equation, which was necessary due to the way in which the code processed the data.

Sets of weather variables were investigated for their ability to predict epidemic status when any or all of the thresholds determined through ROC analysis were reached. All possible combinations of either two or three weather variables were investigated, with a total of 364 combinations. Weather variable sets were excluded where two or more of the variables were calculated from the same raw inputs (Table 2). For each of the 1,000 SiteYear combinations mentioned above, SiteYears were assigned a predicted epidemic status of 1 for each of two scenarios: if any of the thresholds in the weather variable set were reached, and if all of the thresholds were reached. SiteYears were assigned an epidemic status of 0 if the any or all conditions were not met in their respective scenario. This resulted in two epidemic statuses of either 1 or 0 for each SiteYear,

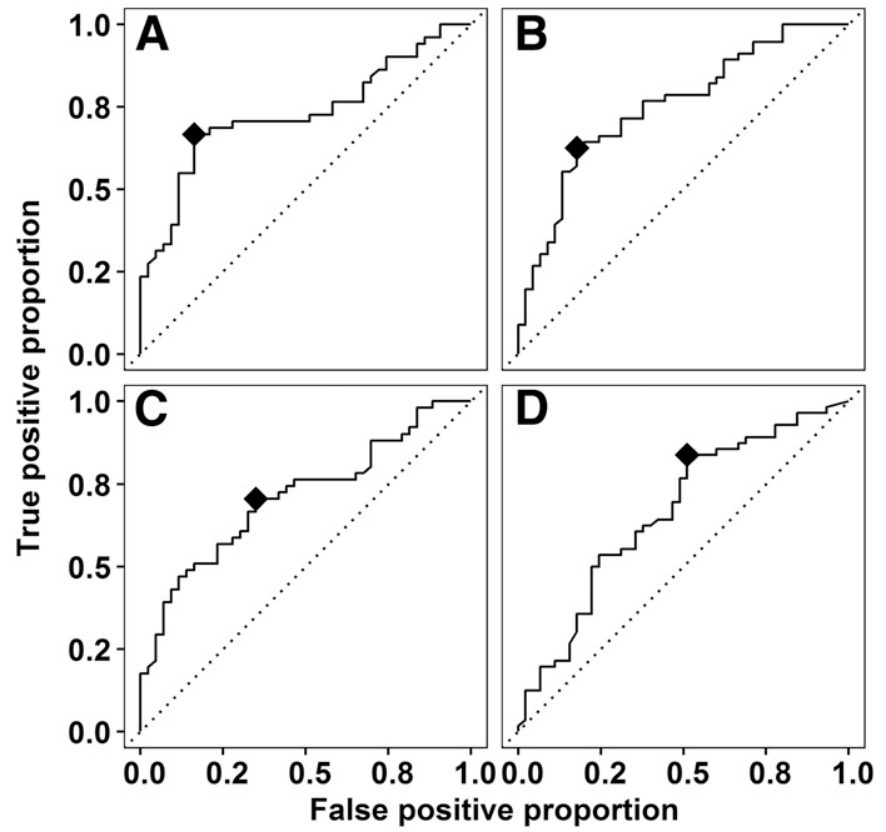

Fig. 4. Example receiver operating characteristic curves for four weather variables: A, RHHoursMD, B, WetThrshMD, C, RHMean, and D, RainLF. Optimum thresholds (diamonds) are the thresholds that maximize true positive proportion and minimize false positive proportion. Curves above the dotted line have better-than-random predictive ability, which is summarized by the area under the curve. Parameters for each curve are shown in Table 4. 
one for the any scenario and one for the all scenario. The $\kappa$ was then calculated for each weather variable set for both the any and all scenarios for each SiteYear combination. The mean $\kappa$ was then calculated across all 1,000 combinations along with the $95 \%$ confidence intervals.

Additional severity thresholds and simplified windowpane analyses. The analyses described above used a BBR severity threshold of $\geq 3 \%$ for case/control determination and weather variables calculated from flowering to harvest in order to create a binary outcome suited to the various analyses. BBR severity thresholds ranging from 1 to $20 \%$ BBR were also used for case/ control determination and the cases and controls were compared using the Kruskal-Wallis test for each severity threshold.

A simplified form of window-pane analysis was also carried out by dividing the fruit development period (flowering to harvest) into 10 intervals for each SiteYear, each with a specified start date and an end date one day before the start date for the following interval, and then calculating weather variables for that period. Intervals starting prior to véraison ( 1 to 6 ), the onset of ripening, were equally spaced between flowering and véraison, with interval 6 ending at véraison. Intervals after véraison ( 7 to 10 ) were equally spaced between véraison and harvest, with interval 10 ending at harvest. More intervals were calculated before véraison than after as the period between flowering and véraison is typically longer than véraison to harvest. Actual dates were specific to each SiteYear. Harvest severity for cases and controls were compared for each time period using the Kruskal-Wallis test.

\section{RESULTS}

Distribution of weather variables. The distribution of values for each weather variable was similar and the majority of variables were skewed toward lower values (Fig. 5). Rainfall-derived variables tended to be skewed toward the lower end, with RainLF having the median normalized value closest to zero. Time-dependent variables (Hours and Days), ThermTime10, and TempMean were the only weather variables with a median normalized value above 0.50 . Thresholds calculated for certain weather variables as described above were $63 \%$ for RHHoursMD and RHHoursTot, $1 \%$ for WetThrshMD, and $17^{\circ} \mathrm{C}$ for ThermTimeThrsh.

Case/control comparisons and logistic regression. Significant differences $(P<0.05)$ were found between the cases and controls for the majority of weather variables using the KruskalWallis test (Table 3). Only five predictor weather variables gave no significant difference $(P>0.05)$ and these were TempMean, TempMin, ThermTime10, RainMD, and RainTot.

Season length (Days and Hours) was found to be a highly significant predictor $(P=0.001)$. Therefore, the 12 time-dependent variables (Days, Hours, ThermTime, ThermTime10, ThermTimeThrsh, WetTot, RHHoursTot, RainTot, BacchusTot, BacchusDryoutTot, BroomeTot, and BroomeDryoutTot) were removed from further analyses (i.e., logistic regression, ROC analysis, skill score analysis, severity threshold analysis, and window-pane analysis) to prevent collinearity with the time component, which may have been the primary driver of the disease epidemic. Mean daily values remained in the analyses as these were independent of season length.

Only one significant model was identified by logistic regression using raw data (see above). Ten significant models were identified by logistic regression using binary data (Table 4 ). The model that was identified using both raw and binary datasets included TempMean and WetMD as explanatory variables and TempMean had a negative coefficient in both cases, indicating higher temperatures inhibited BBR epidemics. Nine of the thirteen weather variables occurred in significant models (BacchusMD, BroomeMD, BacchusDryoutMD, BroomeDryoutMD, RainLF, RHMean, RHHoursMD, TempMean, and WetMD), with no more than two explanatory variables being present in any of the models.
Receiver operating characteristic analysis and skill score testing. The AUC from ROC analysis was significant $(P<0.05)$ for the majority of the weather variables (Table 5), denoting a betterthan-random prediction. Results were similar to those from the Kruskal-Wallis test for weather variables used in both analyses. Only two weather variables (TempMin and RainMD) analyzed using ROC analysis gave nonsignificant AUC values $(P>0.05)$, indicating that their ability to differentiate between cases and controls was no better than random.

Mean kappa scores ( $\kappa$ ) ranged from 0.46 to -0.17 (RHHoursMD and TempMean, respectively) for weather variables in skill score analysis using optimum thresholds from ROC analysis with crossvalidation with 1,000 combinations of training and validation SiteYears (Table 6). The order of weather variables when ranked by $\kappa$ was similar to the order when ranked by either $P$ value using Kruskal-Wallis analysis (Table 3 ) or by $J$ using ROC analysis using all 101 SiteYears (Table 5). Mean optimum thresholds from ROC analysis following cross-validation were highly correlated with those resulting from ROC analysis performed on all SiteYears $(r=$ 0.998).

The highest mean $\kappa$ for any combination of weather variables following skill score analysis was 0.42 when the requirement was for all variable thresholds to be met and 0.38 when the requirement was for any of the variable thresholds to be met (Table 7). No combination of weather variables gave a higher $\kappa$ than

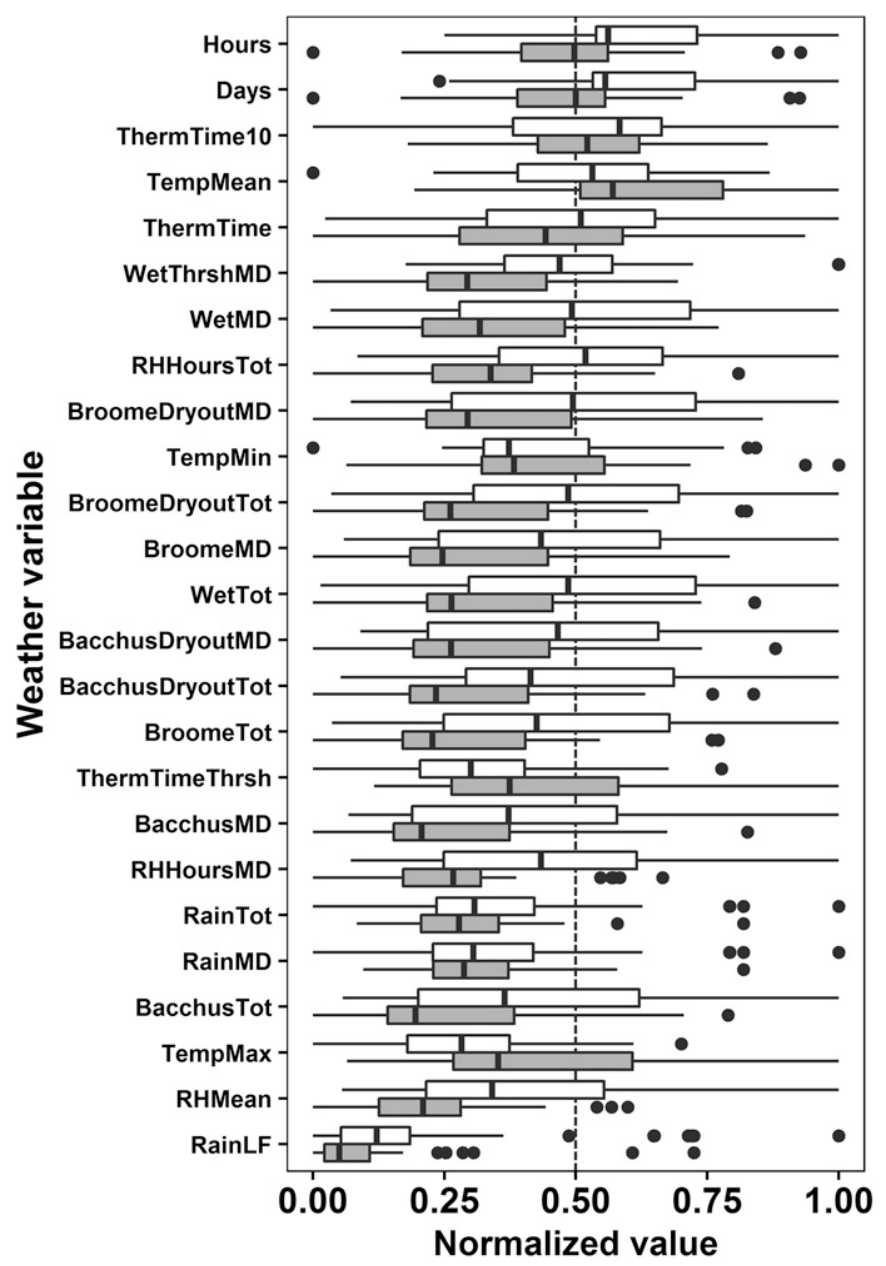

Fig. 5. Summary of weather variables for cases (white) and controls (gray), normalized to a value between 0 and 1 , demonstrating distribution of values $(n=101)$ across all SiteYears (Table 1). Each box represents the first (Q1) and third (Q3) quartile, with a vertical line representing the median. Horizontal lines indicate 1.5 times the interquartile range (IQR; Q3-Q1). Dots represent outliers $(<>1.5 \times \mathrm{IQR})$. Weather variables are sorted by median value. 
TABLE 3. Case and control means for weather variables between flowering (E-L 19) and harvest (E-L 38) across all Site Years ${ }^{\mathrm{a}}$

\begin{tabular}{|c|c|c|c|c|}
\hline Weather variable & Case mean & Control mean & Difference & $P$ value \\
\hline WetThrshMD & 12.6 & 9.9 & 2.6 & $<0.001$ \\
\hline RHMean (\%) & 75.4 & 70.5 & 4.9 & $<0.001$ \\
\hline RHHoursMD & 17.3 & 15.2 & 2.0 & $<0.001$ \\
\hline RHHoursTot & $2,163.1$ & $1,810.6$ & 352.5 & $<0.001$ \\
\hline BacchusDryoutTot & 73.1 & 58.0 & 15.1 & $<0.001$ \\
\hline BroomeDryoutTot & 75.8 & 60.8 & 15.0 & $<0.001$ \\
\hline Days & 125.5 & 118.8 & 6.6 & 0.001 \\
\hline Hours & $3,006.4$ & $2,846.0$ & 160.4 & 0.001 \\
\hline BacchusTot & 44.0 & 33.7 & 10.3 & 0.001 \\
\hline WetTot & 874.0 & 683.6 & 190.4 & 0.002 \\
\hline BroomeTot & 47.4 & 36.7 & 10.7 & 0.002 \\
\hline BacchusDryoutMD & 0.6 & 0.5 & 0.1 & 0.004 \\
\hline RainLF (mm) & 52.7 & 30.1 & 22.5 & 0.005 \\
\hline BroomeDryoutMD & 0.60 & 0.5 & 0.1 & 0.005 \\
\hline WetMD & 6.9 & 5.7 & 1.2 & 0.009 \\
\hline TempMax $\left({ }^{\circ} \mathrm{C}\right)$ & 31.9 & 34.0 & -2.1 & 0.010 \\
\hline BacchusMD & 0.4 & 0.28 & 0.07 & 0.011 \\
\hline BroomeMD & 0.4 & 0.31 & 0.07 & 0.011 \\
\hline ThermTime & $2,143.7$ & $2,073.6$ & 70.1 & 0.027 \\
\hline ThermTimeThrsh & 141.8 & 173.8 & -32.0 & 0.032 \\
\hline TempMean $\left({ }^{\circ} \mathrm{C}\right)$ & 17.1 & 17.51 & -0.4 & 0.061 \\
\hline RainTot (mm) & 288.8 & 236.2 & 52.7 & 0.177 \\
\hline RainMD (mm) & 2.3 & 2.0 & 0.3 & 0.532 \\
\hline ThermTime10 & 889.4 & 887.2 & 2.2 & 0.712 \\
\hline TempMin $\left({ }^{\circ} \mathrm{C}\right)$ & 3.3 & 3.4 & -0.1 & 0.973 \\
\hline
\end{tabular}

a $P$ values are from the Kruskal-Wallis test comparing distributions of cases and controls for each weather variable.

TABLE 4. Significant models resulting from logistic regression performed on binary data using all possible combinations of time-independent weather variables $^{\mathrm{a}}$

\begin{tabular}{llrrrr}
\hline Variable 1 & \multicolumn{1}{c}{ Variable 2 } & \multicolumn{1}{c}{$\beta_{1}$} & $\beta_{2}$ & \multicolumn{1}{c}{$\alpha$} & \multicolumn{1}{c}{ AIC } \\
\hline RainLF & BroomeDryoutMD & 1.538 & 1.880 & -1.488 & 126.9 \\
TempMean & WetMD & -1.029 & 1.986 & 0.543 & 126.5 \\
RainLF & BacchusMD & 1.769 & 1.799 & -1.767 & 126.2 \\
RainLF & BroomeMD & 1.769 & 1.799 & -1.767 & 126.2 \\
RainLF & BacchusMD & 1.769 & 1.799 & -1.767 & 126.2 \\
RainLF & BacchusDryoutMD & 1.678 & 1.760 & -1.747 & 125.4 \\
WetMD & RainLF & 2.385 & 1.918 & -1.858 & 123.4 \\
WetMD & RHMean & 1.649 & 1.055 & -0.624 & 118.4 \\
RHMean & RainLF & 1.503 & 1.711 & -2.102 & 116.9 \\
RHHoursMD & RainLF & 2.447 & 2.258 & -2.766 & 103.2 \\
\hline
\end{tabular}

a Weather variables were calculated for the period between flowering (E-L 19) and harvest (E-L 38) and converted to 0 or 1 based on the thresholds calculated using receiver characteristic analysis (Table 4); $0<$ threshold, $1 \geq$ threshold). Models were selected where the overall model was significant $(P$ $<0.05)$ and all weather variables had a significant effect on the model $(P<$ $0.05)$ and have been ordered by likelihood based on the Akaike information criterion (AIC). $\alpha=$ intercept; $\beta 1$ and $\beta 2=$ coefficients for weather variable 1 and 2 , respectively.
RHHoursMD individually. Eight weather variables were featured in the top 10 combinations for the all scenario (BacchusMD, BroomeMD, BacchusDryoutMD, BroomeDryoutMD, RainLF, RHMean, RHHoursMD, and WetThrshMD) and six were featured in the top 10 combinations for the any scenario (BacchusDryoutMD, BroomeDryoutMD, RainLF, RHHoursMD, WetMD, and WetThrshMD).

Additional severity thresholds and window-pane analyses. Weather variables that were significant for case/control mean separation $(P<0.05)$ with a BBR severity threshold of $3 \%$ were also significant using the majority of the other severity thresholds (Fig. 6). Two weather variables (TempMean and RainMD) were not significant using the $3 \%$ threshold, but were significant using other severity thresholds. RainLF was significant for every severity threshold $(P<0.05)$, while TempMin was not significant $(P>0.05)$ for any severity threshold.

Weather variables that were significant for case/control mean separation $(P<0.05)$ when calculated across the entire season (flowering to harvest) were not always significant when calculated over short intervals during the season (Fig. 7). WetThrshMD was significant $(P<0.05)$ for all intervals except one, while TempMax and RH-derived variables were significant $(P<0.05)$ for earlier and later intervals. WetMD, risk variables, and rainfall-derived variables were mostly significant $(P<0.05)$ for postvéraison intervals. TempMean and TempMin were both only significant $(P<$ 0.05 ) for single intervals.

\section{DISCUSSION}

Automated analyses of aggregated data have not yet been applied widely in studies to identify predictors of plant disease severity. The approach adopted here allowed concurrent assessment of a wide range of statistical methods applied to a large dataset with many variables. Results for the weather-based predictors identified were reinforced by using multiple interrelated types of analysis, some with substantial cross-validation, and many leading to similar conclusions.

Combining ROC analysis with cross-validation and skill score analysis was the approach used in this study with the greatest utility for identifying useful predictive models for BBR epidemics, as it identified weather variables with predictive ability and discriminatory thresholds for those variables with the highest predictive ability, and also tested and evaluated the predictive ability of those thresholds using a simulation of their real-world application (Table 6). The use of ROC analysis has been demonstrated previously in studies of plant disease epidemiology (Beresford et al. 2015; Dewdney et al. 2007; Hughes et al. 1999; Turechek and Wilcox 2005; Yuen et al. 1996). This study enabled ROC analysis, which is computationally-intensive, to be applied to a large dataset with multiple weather variables.

TABLE 5. Receiver operating characteristic analysis results for time-independent weather variables calculated between flowering (E-L 19) and harvest (E-L 38) for all SiteYears ${ }^{\mathrm{a}}$

\begin{tabular}{lccccr}
\hline Weather variable & AUC & Optimum threshold & TPP & FPP & $J$ \\
\hline RHHoursMD & 0.729 & 16.0 & 0.667 & 0.163 & 0.504 \\
WetThrshMD & 0.752 & 12.3 & 0.625 & 0.178 & 0.447 \\
RHMean & 0.711 & 70.7 & 0.706 & 0.349 & 0.357 \\
RainLF & 0.665 & 12.1 & 0.839 & 0.511 & 0.328 \\
WetMD & 0.651 & 8.4 & 0.393 & 0.067 & 0.326 \\
BacchusDryoutMD & 0.667 & 0.59 & 0.482 & 0.156 & 0.326 \\
BroomeDryoutMD & 0.663 & 0.71 & 0.375 & 0.067 & 0.308 \\
BacchusMD & 0.647 & 0.38 & 0.429 & 0.133 & 0.296 \\
BroomeMD & 0.648 & 0.41 & 0.429 & 0.133 & 0.001 \\
TempMax & 0.649 & 32.6 & 0.750 & 0.467 & 0.001 \\
TempMean & 0.609 & 16.9 & 0.482 & 0.222 & 0.283 \\
RainMD & 0.536 & 2.6 & 0.089 & 0.260 \\
TempMin & 0.502 & -0.95 & 0.133 & 0.001 \\
\end{tabular}

a $\mathrm{AUC}=$ area under the curve; TPP $=$ true positive proportion; FPP $=$ false positive proportion; $J=$ Youden's index; and $P$ value $=$ result of Wilcoxon test on AUC. Optimum threshold results in maximum $J$. 
The automated approach used in this study allowed 1,000 random combinations of SiteYears to be used in ROC analysis, crossvalidation and skill-score calculation. This number could have been even higher, as there were $8.75 \times 10^{26}$ possible combinations, although it was found that the $95 \%$ confidence intervals for mean kappa scores ( $\kappa)$ did not decrease substantially above about 500 combinations (data not shown). Although there is some debate over what $\kappa$ means in terms of actual significance, this value indicates the level of agreement between predicted and actual outcomes (McHugh 2012), at least in the medical context where the statistic is commonly applied. In the context of identifying weather predictors associated with plant disease epidemics, $\kappa$ values provide another tool for ranking weather variables for their potential predictive ability. Interestingly, ranking by mean $\kappa$ gave an almost identical weather variable order compared with ranking by $J$ following the initial ROC analysis (Table 3). Additionally, the mean optimum threshold following cross-validation and the optimum threshold resulting from the initial ROC analysis were highly correlated.

While the primary focus was on evaluating the various analyses and the automated way in which they were carried out, there were also some interesting findings in regards to the weather variables themselves. Almost all weather variables demonstrated significance based on both Kruskal-Wallis and ROC analyses. Weather variables that were significant for the Kruskal-Wallis test using a threshold of $3 \%$ BBR severity also tended to be significant using

TABLE 6. Receiver operating characteristic (ROC) analysis results for timeindependent weather variables using 1,000 random combinations of 67 calibration and 34 validation SiteYears ${ }^{\mathrm{a}}$

\begin{tabular}{lcccc}
\hline Weather variable & TPP & FPP & Optimum threshold & $\kappa$ \\
\hline RHHoursMD & 0.657 & 0.193 & 16.0 & 0.45 \\
WetThrshMD & 0.598 & 0.239 & 12.1 & 0.35 \\
RHMean & 0.524 & 0.273 & 72.7 & 0.24 \\
WetMD & 0.429 & 0.186 & 7.70 & 0.23 \\
RainLF & 0.709 & 0.480 & 17.8 & 0.23 \\
BacchusDryoutMD & 0.510 & 0.294 & 0.57 & 0.21 \\
BacchusMD & 0.503 & 0.311 & 0.34 & 0.18 \\
BroomeDryoutMD & 0.440 & 0.248 & 0.64 & 0.18 \\
BroomeMD & 0.455 & 0.273 & 0.39 & 0.17 \\
TempMin & 0.586 & 0.574 & 2.50 & 0.01 \\
RainMD & 0.448 & 0.434 & 2.20 & 0.01 \\
TempMax & 0.356 & 0.494 & 33.0 & -0.13 \\
TempMean & 0.505 & 0.684 & 17.0 & -0.18 \\
\hline
\end{tabular}

a Mean true positive proportion (TPP), false positive proportion (FPP), best weather variable thresholds for case prediction, and kappa $(\kappa)$ scores were calculated for each combination and the means are shown here. All weather variables were calculated for the period from flowering (E-L 19) to harvest (E-L 38). The 95\% confidence intervals for both mean threshold and mean $\kappa$ scores was less than \pm 0.01 in all cases, except for TempMax and RainLF thresholds (0.1 and 0.3 , respectively). Optimum threshold results in maximum Youden's index $(J)$. other severity thresholds ( 1 to $20 \%$ ). These results suggest that the significance is not an artifact of the particular combination of cases and controls, but rather a deeper reflection of a weather variable's influence on the severity of BBR at harvest. The results of the Kruskal-Wallis in particular need to be viewed with caution, as the significance of the test does not necessarily represent good predictive ability, but rather that the distributions of the variables being analyzed are different for cases and controls. Therefore, the Kruskal-Wallis test should only be used to identify variables with potential predictive ability that should then be analyzed further with tests better suited to evaluating predictive ability.

Season length (the period between flowering and harvest) was found to be a significant predictor of BBR epidemics, presumably because the disease had more time to develop in SiteYears where the season was longer, resulting in higher severities. Weather variables accumulated over longer season lengths may appear correlated with disease, which has had a longer period of time to develop, when in fact time is the driving factor. This is important to consider in studies that use data from SiteYears that differ in the timing of crop development. In such circumstances, time-dependent variables should be treated with caution and time should always be investigated for its effects as a predictor of disease.

Weather variables that used thresholds or base values in their calculations, such as surface wetness and thermal time, resulted in lower $P$ values for the difference between cases and controls at the optimum threshold compared with the commonly used threshold. While a lower $P$ value does not necessarily equate to improved predictive power, this was seen across multiple analyses and may suggest that the commonly used thresholds need to be adjusted. The optimum threshold of $1 \%$ for surface wetness was substantially lower than the commonly used threshold of $50 \%$. This result suggests that any surface moisture is conducive to BBR, and that the threshold of $50 \%$ may be underestimating the disease risk.

Variables based on RH and surface wetness were significant and consistent predictors of BBR severity at harvest across the range of analyses applied. Risk variables developed specifically to predict BBR risk (Bacchus and Broome indices) did not outperform the simpler RH- and wetness-related variables. However, these risk variables had higher predictive ability than temperature-derived variables, reflecting the importance of moisture as a driver of the BBR epidemics in the environments studied. RH as a 'raw' weather variable was a reliable predictor and variables derived from $\mathrm{RH}$ gave the greatest predictive ability in the ROC and skill-score analyses. These variables were equal to WetThrshMD and risk models (with dryout periods) in the Kruskal-Wallis analysis, and were present as factors in three of the top 10 models following logistic regression. These findings are consistent with those of previous studies, particularly with respect to RH (Ciliberti et al. 2015; Coertze et al. 2001; Nelson 1951a).

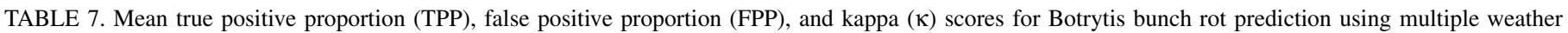

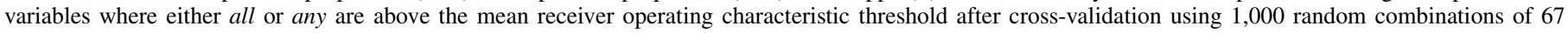
calibration and 34 validation SiteYears using time-independent weather variables ${ }^{\mathrm{a}}$

\begin{tabular}{|c|c|c|c|c|c|c|c|}
\hline \multicolumn{4}{|c|}{ All thresholds reached } & \multicolumn{4}{|c|}{ Any threshold reached } \\
\hline Weather variables & TPP & FPP & $\kappa$ & Weather variables & TPP & FPP & $\kappa$ \\
\hline WetThrshMD \& RHHoursMD & 0.543 & 0.115 & 0.41 & WetThrshMD \& RHHoursMD & 0.702 & 0.314 & 0.38 \\
\hline RHHoursMD \& BacchusMD & 0.440 & 0.068 & 0.36 & WetMD \& RHHoursMD & 0.683 & 0.319 & 0.36 \\
\hline RHHoursMD \& BacchusDryoutMD & 0.444 & 0.071 & 0.36 & RHHoursMD \& RainLF & 0.892 & 0.576 & 0.33 \\
\hline RHHoursMD \& RainLF & 0.456 & 0.091 & 0.35 & WetMD, RHHoursMD \& RainLF & 0.933 & 0.621 & 0.33 \\
\hline WetThrshMD, RHHoursMD \& RainLF & 0.398 & 0.046 & 0.34 & WetMD \& RainLF & 0.866 & 0.555 & 0.32 \\
\hline RHHoursMD \& BroomeMD & 0.410 & 0.063 & 0.33 & RHHoursMD \& BroomeDryoutMD & 0.689 & 0.373 & 0.31 \\
\hline WetThrshMD \& RainLF & 0.429 & 0.095 & 0.32 & $\begin{array}{l}\text { RHHoursMD, RainLF \& } \\
\text { BacchusDryoutMD }\end{array}$ & 0.939 & 0.642 & 0.31 \\
\hline WetMD \& RHHoursMD & 0.379 & 0.053 & 0.31 & RHHoursMD, RainLF \& BroomeDryoutMD & 0.932 & 0.632 & 0.31 \\
\hline RHHoursMD \& BroomeDryoutMD & 0.385 & 0.062 & 0.31 & WetThrshMD \& RHMean & 0.680 & 0.376 & 0.30 \\
\hline WetThrshMD \& RHMean & 0.434 & 0.130 & 0.29 & RainLF \& BacchusDryoutMD & 0.872 & 0.580 & 0.30 \\
\hline
\end{tabular}

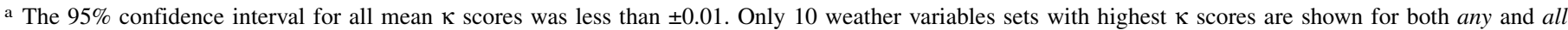
scenarios. 
RHHoursMD also gave the highest mean $\kappa$ for an individual weather variable following skill-score analysis with a value of 0.46 , which would be considered better-than-random, but not high, predictive ability. No combination of weather variables gave an increased $\kappa$ when their thresholds were combined in either the any or all scenarios (Table 7). One of the two variables that gave the highest $\kappa$ after individual variable threshold analysis (RHHoursMD and WetThrshMD) was present in all of the top 10 variable combinations for the all scenario and 8 of the top 10 combinations for the any scenario.

The predominance of $\mathrm{RH}$ as a predictor of disease is consistent with a previous study that used data automation to identify predictors of Fusarium head blight, in which RH-derived variables

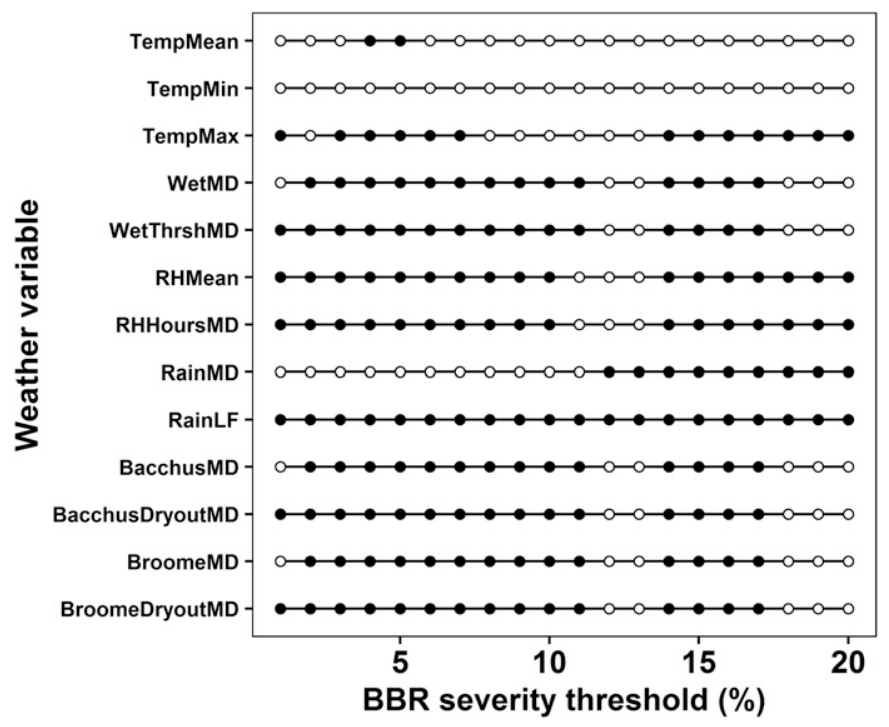

Fig. 6. Results of Kruskal-Wallis tests for time-independent weather variables using various Botrytis bunch rot (BBR) severity thresholds to determine epidemic type (case/control). Black dots indicate a significant difference $(P<$ 0.05 ) between cases and controls for a given severity threshold.

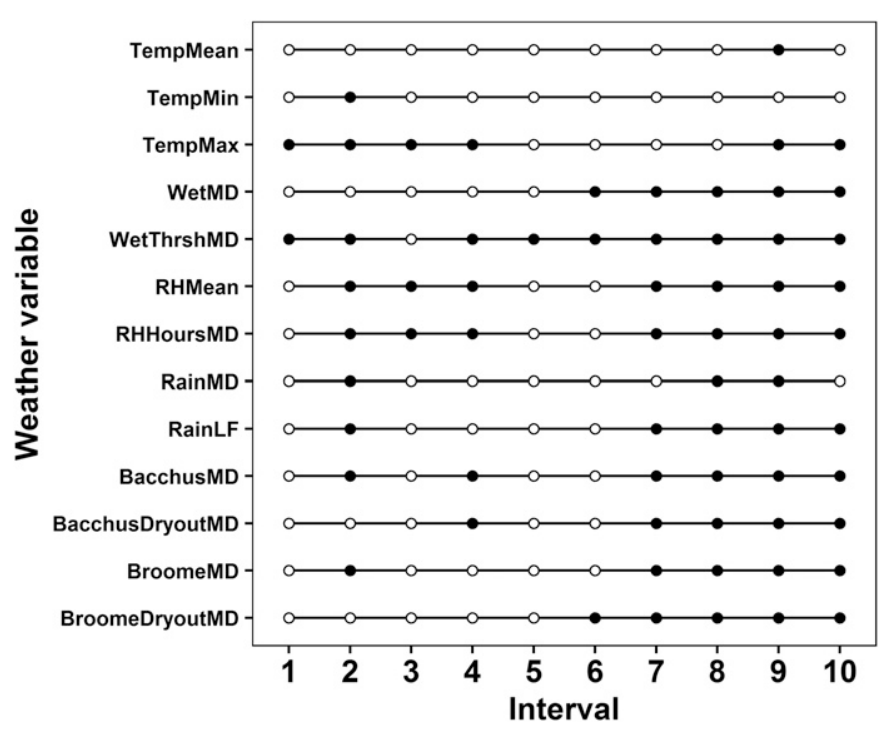

Fig. 7. Results of Kruskal-Wallis tests comparing cases and controls using time-independent weather variables calculated for discrete time intervals throughout the season. Intervals were calculated individually for each SiteYear based on phenology. Intervals 1 to 6 are equally spaced between flowering (E-L 19) and véraison (E-L 35). Intervals 7 to 10 are equally spaced between véraison and harvest (E-L 38). Black dots indicate a significant difference $(P<$ 0.05 ) between cases and controls for a given time period. featured in all 15 significant predictive models (Shah et al. 2013). Variables derived from surface wetness also demonstrated a relatively high level of predictive ability, including the risk models in some analyses. However, surface wetness is not a standard variable recognized by the WMO, so this type of data are not available from many existing weather networks. Relative humidity has been proposed as a proxy for surface wetness in existing disease models because of this lack of standardization and difficulty of calibration (Rowlandson et al. 2015). The increasing availability of accurate $\mathrm{RH}$ sensors and data when combined with the results of this study suggest a need to focus on $\mathrm{RH}$-derived variables for prediction of BBR.

Another significant variable revealed from logistic regression using binary data were RainLF, which was present in 8 of the top 10 significant models (Table 4). This finding is consistent with the perceptions of grape growers who often claim that rain in the weeks before harvest results in higher BBR severity than in dry conditions (Lewis 2017). Other factors involved in the significant regression models using binary data (Table 4) suggest that some combination of preharvest rainfall, temperature and surface wetness could act as a useful predictor of BBR severity at harvest.

Temperature-derived variables were only represented in skill score analysis in the form of the Bacchus or Broome risk models. TempMean was one of the two factors in the only significant model identified by multivariable logistic regression when using the raw weather variables, although this was in combination with WetMD, which is the other input to the Bacchus and Broome indices. TempMean and WetMD also resulted in one of the significant models when using the binary data (Table 4), while the variables based on the Bacchus and Broome indices were significant in many of these models. This suggests that the Bacchus and Broome models combine temperature and wetness in a way that conveys more predictive ability than any combination of the temperature- and wetness-derived variables involved in these analyses.

It was surprising that more significant models were not found through logistic regression using raw, continuous weather variables, even when considering the high chance of type I statistical errors because of the number of models and variables involved in the analyses. This may be an indication that the relationship between weather variables and the development of BBR epidemics is not linear, but it may also be a reflection of the quality of the data, which is discussed below. The advantage of logistic regression was that 8,149 models were evaluated in minutes and then individual models chosen based on the AIC. Of the various information-theoretic approaches that are available, the AIC has been suggested to be best suited for predictive modeling of the type conducted here (Aho et al. 2017). It may be more statistically sound than stepwise model selection (Stanley and Burnham 1998) and was made possible by the automated approach. This method is similar to backward elimination or top-down model selection that results in selection of a single model (Froud 2015), but with the advantage of comparing thousands of models featuring different subsets of timeindependent variables. The main disadvantage of this approach was that it was not practical to evaluate each model individually, although a few were chosen arbitrarily and analyzed manually to ensure coefficients matched, which they did.

Variables derived from rainfall and risk models showed more predictive ability through multivariable logistic regression using binary data than they did through skill score analysis, even though the ROC thresholds used in skill score analyses and to calculate the binary data were essentially the same. This is likely an artifact of the differences in the analyses and demonstrates the potential for predictive ability of weather variables to appear different depending on the analysis used. Combining ROC analysis with logistic regression seems more suited to finding an equation that can be used to calculate degree of risk, whereas skill score analysis can be used to identify a threshold that indicates whether risk of a certain outcome is present or not. Both of these types of output have 
potential value in DSSs; however, their relative use depends on the available management decisions, which is an essential consideration when developing a predictive model.

The potential for adding extra dimensions to the analyses was explored through varying severity thresholds and simplified window-pane analysis. In both cases the analysis was probably not in-depth enough to provide additional insight, although it was adequate to demonstrate the potential of these extra dimensions. The Kruskal-Wallis test was used to evaluate the predictive ability of weather variables using different severity thresholds due to its simplicity, although future analyses could employ ROC analysis in the same manner to provide a series of optimum thresholds for predicting each severity of BBR at harvest. These thresholds could then be combined into a more sophisticated prediction model that could predict harvest severity more accurately. Likewise, windowpane analysis could also be improved through the use of ROC analysis to identify thresholds for weather variables at different stages throughout the season to provide early predictions of BBR epidemics. A more targeted search for weather variables could involve a much higher level of cross-validation for all analyses and more in-depth window-pane analysis to identify predictors relating to specific growth stages or time periods. It may even be possible to perform ROC analysis with a range of severity thresholds and weather variables calculated at different times in the season to generate an array of predictors that could be used to more precisely predict harvest severity at different times during the growing season. While the severity threshold and window-pane analyses performed in this study were not intended to have immediate practical value, they serve to demonstrate how automated analysis makes this sort of multidimensional analysis a possibility.

There are benefits and costs in adopting this automated analytical approach. The initial time required to develop trust in the process by checking the quality of the code, the input data, and validation of information outputs can be substantial. Moreover, the user is removed from actual analyses, especially when using a 'black box' approach like the glmulti package. In short, the data must be of sufficient quality and the code must execute as expected. Data cleaning may be required before the analyses, e.g., error checking and dealing with missing values, and some manual validation is needed after the analyses to increase confidence in the integrity of the data and the system output. The high degree of quality control needed may mean the analyses take longer than the manual alternative. Even so, the automation helps to build researcher insight into how input data are being used by the code, how the code performs the analyses, and the meaning of the information outputs. Once the data and code have been checked, the potential for reusing the analysis framework with minimal manual validation should lead to considerable time-savings.

A feature of both manual and automated data analyses is the dependence on high quality data, including historical weather and disease data; this is exaggerated by automation as the raw data are typically less scrutinized. Surface wetness data, in particular, is notoriously unreliable and nearly impossible to calibrate or verify (Rowlandson et al. 2015). Because of this, optimal threshold calculations in this study may have been incorrect in some instances. The quality of data from any weather sensor may be affected by the type, age, and maintenance of the sensor, but such metadata are rarely stored with the weather data and are therefore not available for use in the analyses (Hunziker et al. 2017). This feature of weather data is important for any disease prediction analysis and requires thorough data checking and confidence in the data source. Weather data collected by national weather-monitoring organizations should be used whenever possible, although the quality of those data should also not be taken for granted and some level of data quality control should always be employed (World Meteorological Organization 2011). It is also important to obtain weather from as close to the source of the disease data as possible, so as to best represent the environment in which that disease developed. Ideally the weather data would be obtained from the same site as the disease data, although a nearby weather station or interpolated weather grid may be suitable in many situations.

The suitability of the disease data for the intended purpose is also very important. The BBR data used in this study was collected from small-plot, replicated trials with a level of scientific rigor appropriate to the purpose of these experiments. Given the reuse of this data for the current study, it is somewhat surprising that so many of the weather variables in this study demonstrated higher than random predictive accuracy, even though none would be considered to have high predictive power. Without further investigation into particular weather variables or targeted experimentation, it is not possible to conclude whether that is because weather does not influence BBR epidemics substantially enough to achieve that high level of prediction, or because the weather and disease data used in the analyses were too imprecise or in some way inappropriate for this purpose.

The availability of increased computing power and advanced data processing techniques are likely to change the way risk models are developed and used and the nature of the output conveyed to growers. In this study, the Bacchus and Broome risk models were used to create specific weather variables rather than implementing them to assess disease risk in the field. Implementation in practice has either involved accumulation of a daily index (Beresford et al. 2012) or graphical presentation of discrete risk periods (Broome et al. 1995; Kim et al. 2007). These implementations provide grape growers with awareness of weather conducive to disease development; however, they provide limited insight on the likely final severity of BBR at harvest. They have also been driven by the need for simple computations. The output of future DSS will still need to relate directly to a management decision and may involve machinelearning techniques that improve the predictions over time (Kaundal et al. 2006) and integrate site-specific knowledge held by the vineyard manager (Evans et al. 2017). Automation of data analyses provides the means to improve empirical models by processing larger and additional datasets to refine the predictive relationships. Use of raw weather variables, rather than calculated indices, reduces the calculations needed to achieve a prediction.

In summary, weather variables were analyzed and compared using a range of rigorous statistical methods, many of which converged on similar predictors. While many of these tests are closely related, and arguably redundant, the benefits of running a wide range of analyses is that there is no need to choose one type of analysis initially and risk neglecting another type of analysis that may yield more useful results. Additionally, identifying the same weather predictor through multiple analyses gives more confidence in its likely predictive ability. There is a substantial risk of type I errors when running such a large number of analyses with such a large number of variables; namely that significance (i.e., the rejection of the null hypothesis) can arise by chance, leading to false conclusions regarding the predictive abilities of weather variables. Therefore, it is important to keep in mind that these sorts of largescale automated analyses are not the final step. They allow a large number of weather variables to be screened with a relatively high level of confidence, but those weather variables should be subjected to more focused analyses, ideally validated using a new data set, and possibly even mechanistically verified through targeted experimentation. Future studies could focus on some of the predictors identified here in different combinations, with various thresholds, and tighter links to phenology in order to further refine their predictive power. Such an intensive predictor investigation will only be possible through the use of data automation, and potentially the incorporation of more advanced machine learning techniques (Mitra 2008). The real power of automating data analyses is the potential to develop and refine analyses in order to answer questions about one data set, and then expand the process to new data sets, such as additional SiteYears and weather variables, or those from other pathosystems. 


\section{ACKNOWLEDGMENTS}

We wish to thank J. Edwards and D. Riches (Department of Primary Industries, Victoria; now DEDJTR), P. Wood, D. Mundy, and R. Agnew (Plant \& Food Research, New Zealand), and C. Henry (Henry Manufacturing) for contributing historical data to this study. We also thank New Zealand Winegrowers, Foundation for Research Science and Technology, Sustainable Farming Fund, Plant \& Food Research, and the former Grape \& Wine Research and Development Corporation, Australia, for co-investing in the collection of the historical data.

\section{LITERATURE CITED}

Aho, K., Derryberry, D., and Peterson, T. 2017. A graphical framework for model selection criteria and significance tests: Refutation, confirmation and ecology. Methods Ecol. Evol. 8:47-56.

Akaike, H. 1974. A new look at the statistical model identification. IEEE Trans. Automat. Contr. 19:716-723.

Andrew, R., and Lupton, T. 2013. Understanding botrytis in New Zealand vineyards. New Zealand Winegrowers, Auckland, NZ.

Beresford, R. M., Evans, K. J., and Hill, G. N. 2012. Botrytis decision support: Online tools for predicting seasonal risk of Botrytis bunch rot. Wine Vitic. J. 27:46-52.

Beresford, R. M., and Kim, K. S. 2011. Identification of regional climatic conditions favorable for development of European canker of apple. Phytopathology 101:135-146.

Beresford, R. M., and Spink, M. 1992. A national disease forecasting system for apple black spot (Venturia inaequalis) in New Zealand. Acta Hortic. 285-296.

Beresford, R. M., Tyson, J. L., and Henshall, W. R. 2016. Development and validation of an infection risk model for bacterial canker of kiwifruit, using a multiplication and dispersal concept for forecasting bacterial diseases. Phytopathology 107:184-191.

Beresford, R. M., Wood, P. N., and Fisher, B. M. (2015) Use of receiver operating characteristic curve analysis for calibrating plant disease risk forecasting models. Pages 3-43 in: The Plant Protection Data Tool Box. R. M. Beresford, K. J. Froud, J. M. Kean, and S. P. Worner, eds. New Zealand Plant Protection Society.

Broome, J. C., English, J. T., Marois, J. J., Latorre, B. A., and Aviles, J. C. 1995. Development of an infection model for Botrytis bunch rot of grapes based on wetness duration and temperature. Phytopathology 85:97-102.

Calcagno, V. 2013. glmulti: Model selection and multimodel inference made easy. $\mathrm{R}$ package version 1.0.7. https://cran.r-project.org/web/packages/ glmulti/index.html

Ciliberti, N., Fermaud, M., Languasco, L., and Rossi, V. 2014. Influence of fungal strain, temperature, and wetness duration on infection of grapevine inflorescences and young berry clusters by Botrytis cinerea. Phytopathology 105:325-333.

Ciliberti, N., Fermaud, M., Roudet, J., and Rossi, V. 2015. Environmental conditions affect Botrytis cinerea infection of mature grape berries more than the strain or transposon genotype. Phytopathology 105:1090-1096.

Coakley, S. M., McDaniel, L. R., and Shaner, G. 1985. Model for predicting severity of Septoria tritici blotch on winter wheat. Phytopathology 75: 1245-1251.

Coertze, S., Holz, G., and Sadie, A. 2001. Germination and establishment of infection on grape berries by single airborne conidia of Botrytis cinerea. Plant Dis. 85:668-677.

Cohen, J. 1960. A coefficient of agreement for nominal scales. Educ. Psychol. Meas. 20:37-46.

Coombe, B. G. 1995. Adoption of a system for identifying grapevine growth stages. Aust. J. Grape Wine Res. 1:104-110.

Cox, D. R. 1958. The regression analysis of binary sequences. J. R. Stat. Soc. B 20:215-242.

Dewdney, M. M., Biggs, A. R., and Turechek, W. W. 2007. A statistical comparison of the blossom blight forecasts of MARYBLYT and cougarblight with receiver operating characteristic curve analysis. Phytopathology 97:1164-1176

Edwards, J., Riches, D., Evans, K., Beresford, R. M., Hill, G. N., Wood, P. N., and Mundy, D. C. 2009. The need for a risk-based approach to botrytis management. The Australian \& New Zealand Grapegrower \& Winemaker Annual Technical Issue 6-9

Evans, K. J. 2013. Assessing and managing disease-affected fruit in the vineyard: The Australian experience. Pages 11-19 in: ASVO-Making the Best Out of Difficult Vintages: Managing Sub-Optimal Fruit in the Winery, Adelaide, SA, Australia.

Evans, K. J., Terhorst, A., and Kang, B. H. 2017. From data to decisions: Helping crop producers build their actionable knowledge. Crit. Rev. Plant Sci. 36:71-88.
Froud, K. J. 2015. Two case studies using observational study designs and multivariable analysis to investigate kiwifruit bacterial canker in New Zealand. Pages 121-137 in: The Plant Protection Data Toolbox. R. M. Beresford, K. J. Froud, J. M. Kean, and S. P. Worner, eds. The New Zealand Plant Protection Society, Auckland, NZ.

Gent, D. H., Mahaffee, W. F., McRoberts, N., and Pfender, W. F. 2013. The use and role of predictive systems in disease management. Annu. Rev. Phytopathol. 51:267-289.

González-Domínguez, E., Caffi, T., Ciliberti, N., and Rossi, V. 2015. A mechanistic model of Botrytis cinerea on grapevines that includes weather, vine growth stage, and the main infection pathways. PLoS One 10: $\mathrm{e} 0140444$.

Gouache, D., Leon, M. S., Duyme, F., and Braun, P. 2015. A novel solution to the variable selection problem in Window Pane approaches of plant pathogen-climate models: Development, evaluation and application of a climatological model for brown rust of wheat. Agric. For. Meteorol. 205: 51-59.

Greiner, M., Pfeiffer, D., and Smith, R. D. 2000. Principles and practical application of the receiver-operating characteristic analysis for diagnostic tests. Prev. Vet. Med. 45:23-41.

Hanley, J. A., and McNeil, B. J. 1982. The meaning and use of the area under a receiver operating characteristic (ROC) curve. Radiology 143:29-36.

Heidke, P. 1926. Berechnung des erfolges und der güte der windstärkevorhersagen im sturmwarnungsdienst (Measures of success and goodness of wind force forecasts by the gale-warning service). Geogr. Ann. 8:301-349.

Hijmans, R. J. 2016. geosphere: Spherical Trigonometry. R package version 1.5-5. https://cran.r-project.org/web/packages/geosphere/index.html

Hill, G. N., Beresford, R. M., and Evans, K. J. 2010. Tools for accurate assessment of Botrytis bunch rot (Botrytis cinerea) on wine grapes. N. Z. Plant Prot. 63:174-181.

Hill, G. N., Evans, K. J., and Beresford, R. M. 2014. Use of nitrate nonutilising (nit) mutants to determine phenological stages at which Botrytis cinerea infects wine grapes causing botrytis bunch rot. Plant Pathol. 63: $1316-1325$.

Hughes, G. 2012. Applications of Information Theory to Epidemiology. American Phytopathological Society, St. Paul, MN.

Hughes, G., McRoberts, N., and Burnett, F. J. 1999. Decision making and diagnosis in disease management. Plant Pathol. 48:147-153.

Hunziker, S., Gubler, S., Calle, J., Moreno, I., Andrade, M., Velarde, F., Ticona, L., Carrasco, G., Castellón, Y., Oria, C., Croci-Maspoli, M., Konzelmann, T., Rohrer, M., and Brönnimann, S. 2017. Identifying, attributing, and overcoming common data quality issues of manned station observations. Int. J. Climatol. 37:4131-4145.

Hyvarinen, O. 2014. A probabilistic derivation of Heidke skill score. Weather Forecast. 29:177-181.

Kaundal, R., Kapoor, A. S., and Raghava, G. P. S. 2006. Machine learning techniques in disease forecasting: A case study on rice blast prediction. BMC Bioinformatics 7:485.

Kim, K. S., Beresford, R. M., and Henshall, W. R. 2007. Prediction of disease risk using site-specific estimates of weather variables. N. Z. Plant Prot. 60: $128-132$

Kriss, A. B., Paul, P. A., and Madden, L. V. 2010. Relationship between yearly fluctuations in Fusarium head blight intensity and environmental variables: A window-pane analysis. Phytopathology 100:784-797.

Kruskal, W. H., and Wallis, W. A. 1952. Use of ranks in one-criterion variance analysis. J. Am. Stat. Assoc. 47:583-621.

Lewis, O. 2017. Marlborough wine companies bring in grapes ahead of ' $D$ Day' rain event threatening crop losses. The Marlborough Express. https:// www.stuff.co.nz/business/farming/agribusiness/91185174/marlboroughwine-companies-bring-in-grapes-ahead-of-dday-rain-event-threateningcrop-losses

Mann, H. B., and Whitney, D. R. 1947. On a test of whether one of two random variables is stochastically larger than the other. Ann. Math. Stat. 18: 50-60.

McHugh, M. L. 2012. Interrater reliability: the kappa statistic. Biochem. Med. (Zagreb) 22:276-282.

Mitra, S. 2008. Introduction to machine learning and bioinformatics. CRC Press, Boca Raton, FL.

Mourtzinis, S., Rattalino Edreira, J. I., Conley, S. P., and Grassini, P. 2017. From grid to field: Assessing quality of gridded weather data for agricultural applications. Eur. J. Agron. 82:163-172.

Nair, N. G., and Allen, R. N. 1993. Infection of grape flowers and berries by Botrytis cinerea as a function of time and temperature. Mycol. Res. 97: 1012-1014.

Nelson, K. E. 1951a. Effect of humidity on infection of table grapes by Botrytis cinerea. Phytopathology 41:859-864.

Nelson, K. E. 1951b. Factors influencing the infection of table grapes by Botrytis cinerea (Pers.). Phytopathology 41:319-326. 
R Core Team. 2016. R: A language and environment for statistical computing. R Foundation for Statistical Computing, Vienna, Austria. https://www.R-project.org/

R Studio Team. 2016. RStudio: Integrated Development for R. RStudio, Boston, MA. https://www.rstudio.com/.

Robin, X., Turck, N., Hainard, A., Tiberti, N., Lisacek, F., Sanchez, J.-C., and Muller, M. 2011. pROC: An open-source package for $\mathrm{R}$ and $\mathrm{S}+$ to analyze and compare ROC curves. BMC Bioinformatics 12:77.

Rowlandson, T., Gleason, M., Sentelhas, P., Gillespie, T., Thomas, C., and Hornbuckle, B. 2015. Reconsidering leaf wetness duration determination for plant disease management. Plant Dis. 99:310-319.

Scherm, H., Thomas, C. S., Garrett, K. A., and Olsen, J. M. 2014. Metaanalysis and other approaches for synthesizing structured and unstructured data in plant pathology. Annu. Rev. Phytopathol. 52:453-476.

Shah, D. A., Molineros, J. E., Paul, P. A., Willyerd, K. T., Madden, L. V., and De Wolf, E. D. 2013. Predicting Fusarium head blight epidemics with weather-driven pre- and post-anthesis logistic regression models. Phytopathology 103:906-919.

Shorthouse, D. P. 2017. SimpleMappr, an online tool to produce publicationquality point maps. http://www.simplemappr.net

Shtienberg, D. 2013. Will decision-support systems be widely used for the management of plant diseases? Annu. Rev. Phytopathol. 51:1-16.

Stanley, T. R., and Burnham, K. P. 1998. Information-theoretic model selection and model averaging for closed-population capture-recapture studies. Biometrical J. 40:475-494.
Student. 1908. The probable error of a mean. Biometrika 6:1-25.

Turechek, W. W., and Wilcox, W. F. 2005. Evaluating predictors of apple scab with receiver operating characteristic curve analysis. Phytopathology 95: 679-691.

Wickham, H. 2009. ggplot2: Elegant Graphics for Data Analysis. SpringerVerlag, New York.

Wickham, H. 2016. tidyverse: Easily Install and Load 'Tidyverse' Packages. https://cran.r-project.org/web/packages/tidyverse/index.html

Wilcoxon, F. 1945. Individual comparisons by ranking methods. Biom. Bull. $1: 80-83$.

World Meteorological Organization. 2011. Guide to Climatological Practices. World Meteorological Organization, Geneva, Switzerland.

World Meteorological Organization. 2014. Guide to Meteorological Instruments and Methods of Observation: (CIMO Guide). World Meteorological Organization, Geneva, Switzerland.

Xie, Y. 2016. knitr: A General-Purpose Package for Dynamic Report Generation in $\mathrm{R}$. R package version 1.14. https://yihui.name/knitr/

Youden, W. J. 1950. Index for rating diagnostic tests. Cancer 3:32-35.

Yuen, J., Twengstrom, E., and Sigvald, R. 1996. Calibration and verification of risk algorithms using logistic regression. Eur. J. Plant Pathol. 102:847-854

Zweig, M. H., and Campbell, G. 1993. Receiver-operating characteristic (ROC) plots: A fundamental evaluation tool in clinical medicine. Clin. Chem. 39:561-577. 